\title{
APORTES DE LOS ESTUDIOS DE GÉNERO A LAS CIENCIAS SOCIALES
}

\section{The contributions of Gender Studies to the Social Sciences}

\section{LORETO REBOLLEDO *}

\section{Resumen}

Este artículo presenta un recorrido de los aportes y trayectorias de los estudios de género para el caso chileno, a partir de la revisión de las principales transformaciones en este campo a nivel mundial y su influencia sobre América Latina. A partir de la década de 1970, las ciencias sociales entran en una crisis donde no solo se ponen en entredicho los grandes paradigmas explicativos, sino también los modos de acceso y construcción del conocimiento. Las visiones universalistas comienzan a ser cuestionadas ante la aparición de las políticas de la identidad tras el debate generado por los movimientos sociales y sus reivindicaciones específicas, entre ellos, el movimiento feminista de los países del primer mundo, cuyas críticas a la influencia de la variable de género en la construcción del conocimiento dan origen a los estudios de la mujer, antecedente directo de lo que conocemos hoy como estudios de género. Estos estudios, construidos a partir de la permanente crítica y revisión por parte de las/os investigadoras/es del campo, han aportado nuevas miradas, temas y formas de abordaje interdisciplinario, contribuyendo así a la revitalización de las ciencias sociales en el mundo, pero, sobre todo en el contexto latinoamericano. En este artículo se da cuenta de estos aportes, mostrando el desarrollo de los estudios de mujer y género en Chile y, revisando el contexto general en que surgieron; su instalación en el país; sus principales aportes y los desafíos que enfrentan en la actualidad.

Palabras Clave: Estudios de género, Antropología, Chile

\section{Abstract}

This article presents an overview of the contributions and trajectories of gender studies for the Chilean case, from a revision of the main changes in this field in a world level and their influence on Latin America. From the seventies, the social sciences enter into a crisis where not only call into question the more important explanatory paradigms, but also modes of access and knowledge construction. Universalist visions begin to be challenged by the emergence of identity politics, after the debate generated by social movements and their specific demands. One of the social movements that breaks in scene in

\footnotetext{
* Antropóloga y periodista, Doctora en Historia, académica del Instituto de Comunicación e Imagen-ICEI, y del Centro Interdisciplinario de Estudios de Género, Facultad de Ciencias Sociales, Universidad de Chile.

Correo electrónico: mareboll@u.uchile.cl
} 
those moments was the feminist movement of the first world countries, of which criticism of the influence of the gender variable in building knowledge gives origin to women's studies, direct predecessor of what we know today as Gender Studies. The Gender Studies, constructed from continuing criticism and revision by the researchers of the field, have provided new perspectives, issues and ways to address them in an interdisciplinary way, contributing to the revitalization of the social sciences in the world but especially in the Latin American context. This article accounts for these contributions, showing the development of Women Studies and Gender Studies in Chile, through a review of the overall context in which they arose, its consolidation in the country and the challenges facing today.

Key words: Gender studies, Anthropology, Chile.

\section{Introducción}

Quizás el aporte más evidente de los estudios de género es el rechazo al supuesto que confunde lo humano con lo masculino, el cual desde hace dos mil años ha constituido la base de los paradigmas y se ha infiltrado tanto en la teoría sustantiva de nuestras disciplinas como en los terrenos cotidianos (Tarrés, 1999:19)

Tradicionalmente lo humano fue entendido como lo masculino y ello tuvo implicancias de diverso orden, entre otras cosas dejar fuera de la historia, las ciencias, las artes, a la mitad de la humanidad. Sin embargo, como invisibilidad no es sinónimo de ausencia este déficit se hizo evidente en la década de los setenta del pasado siglo, cuando los movimientos feministas comenzaron a denunciar la subordinación de la que habían sido víctimas las mujeres y a desarrollar estudios empíricos que demostraban los aportes femeninos a la economía y la cultura. Así, desde los inicios existió un fuerte vínculo entre política y academia, los estudios de mujer y género surgen y se desarrollan a partir de esa relación, buscando dar respuesta a la incomodidad del no lugar al que habían sido relegadas las mujeres por las disciplinas, y de paso interpelándolas. En un primer momento, los estudios de la mujer, estrechamente ligados al feminismo político, se orientaron a dar cuenta sobre el modo en que operaba la subordinación de las mujeres, entendiéndola como universal y a completar el vacío de información existente sobre las actividades de las mujeres en diferentes áreas y sociedades. Paralelamente, buscaron desmontar el aparataje que posibilitó entender lo humano como sinónimo de lo masculino, invisibilizando en sus análisis a las mujeres y, para ello, se concentraron en la revisión de las distintas disciplinas. Una apuesta de este tipo implicaba una subversión de los modos de construir el conocimiento, donde no era suficiente llenar las lagunas existentes ya que el desafío era tratar de cambiar la verdadera naturaleza de lo que se considera conocimiento.

El cuestionamiento a las disciplinas realizado desde el feminismo abarcó los paradigmas establecidos, las prácticas y las teorías. En ese proceso fueron socavadas algunas "verdades" establecidas, por ejemplo, en relación a la objetividad y neutralidad de la ciencia. Los estudios de la mujer y de género, a través de la revisión de numerosas 
investigaciones realizadas dentro de los cánones tradicionales de las disciplinas - como la historia, antropología, sociología, entre otras-, pudieron demostrar que el conocimiento es situado, que no hay neutralidad en el sujeto que conoce, que el conocimiento es construido desde y en función de las relaciones sociales que el sujeto cognoscente establece. Como señala Gloria Bonder:

"La crítica feminista ha puntualizado el carácter situado del conocimiento, la parcialidad de todas las afirmaciones, la íntima relación entre saber y poder, en definitiva ha colocado a las grandes narrativas en el incómodo contexto de la política, retirándolas del "confortable dominio de la epistemología". En este sentido y como dice Giroux, el feminismo ha sostenido en clave de género, una pregunta fundamental frente al saber instituido: ¿Quién habla en esa teoría, bajo qué condiciones sociales, económicas y políticas formula ese discurso; para quién y cómo ese conocimiento circula y es usado en el marco de relaciones asimétricas de poder" (Bonder, 1999:30).

En este sentido, es de destacar el aporte de la antropología feminista al proponer los conceptos de etno y androcentrismo ${ }^{1}$ como manera de entender la forma en que operaron los antropólogos en el momento de realizar sus investigaciones. Más allá de algunos esfuerzos individuales de mujeres antropólogas, como Margaret Mead y otras que la precedieron (cfr. Stolcke), en general, la antropología pecó de ambos. Los antropólogos en su trabajo de terreno, fueron incapaces de desprenderse de los prejuicios que traían de sus propias sociedades occidentales y tendieron a ver a las mujeres de las sociedades estudiadas, solo como elementos de intercambio o parte integrante de los sistemas de parentesco.
En general, eran otros hombres (los informantes locales) quienes daban cuenta -si es que se les consultaba- de lo que hacían las mujeres. Esto provocó una carencia de datos sobre la vida cotidiana de las mujeres o bien visiones muy empobrecidas respecto a lo que ellas hacían y aportaban en sus respectivas comunidades, que fue denunciada por las feministas y que las antropólogas trataron de resolver buscando recoger el punto de vista de las mujeres de las sociedades estudiadas. Ya a mediados de la década de los setenta comienzan a difundirse publicaciones de antropólogas, como Michelle Rosaldo, Rayna Reiter y otras, que buscaban responder a la pregunta sobre la causa de su opresión universal. Para ello, se volcaron a buscar los elementos que eran compartidos en todas las sociedades por las mujeres. Entre las respuestas que se propusieron estaban las consecuencias sociales de la maternidad; la dicotomía naturaleza-cultura, donde la segunda domina a la primera y las mujeres, dadas sus características biológicas se asocian a la naturaleza (Ortner, 1979); la otra explicación fue la dicotomía público-privado que mostrarían todas las sociedades, donde los hombres dominan lo público y las mujeres quedan relegadas a lo privado. Otras explicaciones a la opresión de las mujeres, propuestas por antropólogas marxistas, se buscaron en la aparición del capitalismo y, en las sociedades precapitalistas como efecto del colonialismo (Mona \& Leacock, 1980) o del paso de la caza y recolección a la agricultura. Como una manera de superar la falta de información sobre las mujeres, se produjo un incremento importante de los estudios empíricos que daban cuenta de sus trabajos productivos y reproductivos, sus prácticas religiosas y festivas, etc., los que de 
a poco fueron mostrando que había variaciones importantes en el lugar asignado a las mujeres en las diversas sociedades y ponían por tanto en cuestión la validez de la universalidad de su opresión.

Paralelamente, en la década de los ochenta, el movimiento feminista del tercer mundo comienza a hacerse escuchar y, junto a la protestas de mujeres negras de países desarrollados, cuestionan al feminismo occidental acusándolo de etnocentrismo, al proyectar sus propias experiencias de discriminación al conjunto de mujeres del planeta, a las "otras" 2 . Por otra parte, se hace evidente, a partir de la recolección de datos empíricos la existencia de diferencias de poder entre las propias mujeres. Poco a poco, se comienza a abrir paso el concepto de género, que descansa sobre la premisa que en todas las sociedades el sexo es uno de los criterios de clasificación de las personas, sin embargo, las relaciones entre hombres y mujeres varían entre las diversas sociedades de acuerdo a definiciones culturales e históricas y al entrelazamiento con otras variables: clase, etnia, raza, edad, orientación sexual.

Posteriormente, las mujeres del tercer mundo y las mujeres negras en Estados Unidos ${ }^{3}$, comenzaron a revisar críticamente -a la luz de sus propias experiencias de vida- los conceptos e interpretaciones planteados por las feministas del primer mundo que habían impulsado los estudios de la mujer. Así, ya no solo la subordinación universal es puesta en jaque, otras categorías son examinadas, por ejemplo, desde las mujeres negras de Norteamérica se muestra la limitación de las categorías público-privado, resultantes de la división sexual del trabajo para dar cuenta de su situación social, ya que para la mayoría de ellas lo público actuaba como espacio de opresión, por razones de clase y raza y, lo privado, era lugar de afecto y contención. Es decir, operaba de manera inversa a lo planteado por los estudios de la mujer y feministas "blancas".

A partir de estas miradas críticas afloran las diferencias entre mujeres, que muchas veces pueden ser mayores que las existentes con los hombres de su mismo grupo ${ }^{4}$. Las variables, clase social, raza, etc., aparecen intersectándose con el género evidenciando que la dominación/ opresión es multidimensional y, en la medida en que actúan de manera simultánea, no es posible plantear explicaciones solo a partir de una de esas variables (ser mujer) y, dejando de lado, otros elementos que conforman la vida.

Aunque muchas veces tienden a ser confundidos, es importante considerar que hay una diferencia importante entre los estudios de la mujer -donde se evidencia claramente una visión monolítica y categórica de las mujeres como un colectivo universalmente oprimido dada su identidad de género, sin considerar las otras variables que relativizan, sitúan y condicionan sus vidas-y los estudios de género, donde se ha intentado situar cultural, social política e históricamente, la vida de las mujeres y los hombres, viendo cómo se entrelazan en sus identidades múltiples diferencias: sexuales, de clase, raza, edad y etnia, que inciden en el mayor o menor poder con que cuentan.

El desarrollo de los estudios de género, permitió pasar de una visión unívoca de la mujer, subordinada universalmente, a una donde hombres y mujeres detentan identidades en que convergen diferentes factores $y$, donde las subordinaciones, son relativizadas en la convergencia de estos, sin operar de manera unívoca y unidireccional entre hombres y mujeres. 
La antropóloga mexicana Marta Lamas, sostiene:

\begin{abstract}
"Siguiendo a Deleuze y Guattari hemos tomado la identidad como una articulación que conecta multiplicidades insertas en una variedad de órdenes discursivos (...) podemos pensar las identidades como anudamientos provisorios en entretejidos históricamente contingentes, anudamientos que son desanudados y vueltos a anudar" (Lamas, 1996: IX).
\end{abstract}

Ello obliga a analizar a los sujetos en sus respectivos contextos y en sus diferentes relaciones sociales. A partir de este ejercicio, es posible por ejemplo, ampliar la imagen de las mujeres del tercer mundo, construida por el feminismo occidental como una víctima pasiva y subordinada de los hombres, sumida en la pobreza y la indefensión, por la imagen de mujeres-sujeto con capacidad de agencia, de resistencia y de empoderarse, pese a sus carencia de poder y las limitaciones materiales de sus vidas.

La introducción de la noción de género en los análisis sociales, trajo consigo una serie de rupturas epistemológicas, respecto a las maneras en que se había entendido la posición de las mujeres en las distintas sociedades humanas. En primer lugar, hizo énfasis en los aspectos culturales más que en los biológicos, además aportó la idea de variabilidad, dado que al ser mujer $u$ hombre un constructo cultural, sus definiciones varían entre unas culturas y otras; por otra parte, plantea la necesidad de lo relacional: el género como construcción social de las diferencias sexuales, alude a las distinciones entre femenino y masculino $y$, por ende, a las relaciones entre ellos; pone en escena el principio de la multiplicidad y convergencia de elementos, que constituyen la identidad del sujeto. De este modo, se propone comprender, a los sujetos mujeres y hombres, no solo desde uno de sus perfiles (el género) sino desde las categorías que habitan en ellos simultáneamente y, que van a modelar y especificar, su ser femenino o masculino. Por último, emerge la idea de posicionamiento: un análisis de género, supondrá el estudio del contexto en el que se dan las relaciones de género de hombres y mujeres y, de la diversidad de posiciones que ellos ocuparán, sobre todo en las sociedades complejas (cfr. Montecino y Rebolledo, 1995).

\section{Contexto del desarrollo de los estudios de la mujer y género}

El surgimiento de los estudios de mujer y género en Chile y, su posterior institucionalización, sigue derroteros similares a los otros países de la región. En América Latina, al igual que en otros lugares del mundo ${ }^{5}$, los estudios de género y los estudios de la mujer surgieron vinculados estrechamente a los movimientos feministas. Mientras que en los países del primer mundo, el feminismo irrumpió en la década de los setenta, en América Latina serán los años ochenta los que verán emerger un poderoso movimiento de mujeres $y$, una multiplicidad de organizaciones de mujeres y feministas, en un contexto en que las dictaduras y gobiernos autoritarios se habían entronizado en parte importante del continente.

En un primer momento, fueron los estudios de la mujer los que nacieron de la mano de los movimientos feministas. Las ONG, centros de estudio de carácter privado y, el trabajo voluntario de mujeres académicas en algunas universidades, fueron las principales impulsoras de este tipo de estudios. Sin embargo, su inserción institucional varía de acuerdo a la situación política de los diferentes países. 
En aquellos países con presencia de gobiernos autoritarios o dictatoriales -Argentina, Uruguay, Paraguay, Argentina, Colombia, Perú, Chile y Puerto Rico-, surgen en centros independientes y ONG y, solo logran institucionalizarse al cambiar las condiciones políticas y democratizarse las universidades (Bonder,1998).

Algunos de los centros de la mujer y ONG de mujeres, desarrollaron un papel importante al realizar estudios sobre diversas dimensiones de las vidas de las mujeres, a la par que se llevaba adelante, una denuncia pública de su situación y se promovía el trabajo de autoconciencia entre las propias mujeres, a partir de la realización de talleres y encuentros. Inicialmente se trataba de trabajos más bien descriptivos, donde se buscaba mostrar la participación de las mujeres en la educación, el trabajo, la salud, a partir del uso de datos estadísticos, pero también con mucho relato testimonial, buscando dar voz a aquellas que habían sido silenciadas por la historia e invisibilizadas por las otras disciplinas.

Inicialmente, los conceptos y categorías utilizados para analizar la realidad femenina eran tomados prestados de las feministas del primer mundo, sin mayores intentos de realizar una teorización propia o de poner una mirada crítica respecto a ellos. Básicamente, pretendían dar cuenta de la situación de desventaja de las mujeres en diversos ámbitos $\mathrm{y}$, hubo un periodo, durante el cual se tendieron a confundir los conceptos de mujer y género. Como consigna Stolke:

"La noción de género se ha convertido en una especie de término académico sintético, que aunque enfatice la construcción social de las identidades de mujeres y hombres, con frecuencia es simplemente mal utilizada como sinónimo culturalista de sexo" (Stolke: s.f.).
Sin embargo, a poco andar, diversas mujeres iniciaron una rica reflexión desde la realidad propia que permitió enriquecer los debates y aportar a la teoría desde una perspectiva latinoamericana, a partir de un florecimiento de las publicaciones y revistas. México, en tanto lugar de exilio de mujeres de Sudamérica, se transformó en un importante centro irradiador de ideas e impulsor de congresos, seminarios y encuentros que colaboraron al desarrollo de los estudios de mujer y género en la región ${ }^{6}$.

En México, Brasil, Venezuela, Costa Rica y República Dominicana, los estudios de la mujer y género surgieron al interior de los centros académicos universitarios, gracias a la militancia feminista de algunas de las académicas (cfr. Bonder, 1998). Aunque ya en 1975 , como resultado de los debates de la Primera Conferencia Internacional de la Mujer, las ideas feministas habían ingresado al ámbito de la educación superior. Sin embargo, la institucionalización en espacios universitarios académicos, en la mayor parte de los países, se produce en la década de los ochenta, si bien este proceso fue más tardío en los países que tenían intervenidas sus universidades. Estos estudios de mujeres eran hechos por mujeres, siguiendo un camino similar al que ocurrió en los países del primer mundo. Los estudios de la mujer, en los inicios de su desarrollo, aparecen fuertemente vinculados a lo político y, las temáticas abordadas, son las mismas que las de los movimientos feministas, aunque no se reducen solo a ello.

El paso de la denuncia política y las reivindicaciones de igualdad del movimiento feminista, a su institucionalización, se dio gracias a que las académicas feministas fueron capaces de articular un discurso que, pese a las suspicacias y reticencias de sus colegas hombres, logró poco a 
poco legitimarse en las universidades; así, debates que antes eran propios de los grupos de reflexión feministas, ingresaron a las aulas y tuvieron un impacto importante en las disciplinas tradicionales. La docencia, la investigación y las numerosas publicaciones, dan cuenta de la fecundidad del aporte hecho por los estudios de género.

\section{Los estudios de género en Chile}

En Chile, la institucionalización en espacios universitarios de los estudios de la mujer y género, recién se produce en los noventa, cuando retorna la democracia y las universidades dejan de estar vigiladas. Sin embargo, los orígenes de los estudios de mujer y género se remontan a los años ochenta, momento en que la conciencia de género va surgiendo de la mano de la lucha contra la dictadura a partir de la práctica cotidiana y, la reflexión de mujeres participantes en organizaciones y grupos de mujeres de diverso tipo: agrupaciones de derechos humanos, organizaciones económicas populares, colectivos de mujeres, comedores infantiles y ollas comunes. Paralelamente a ello, grupos de estudios de mujeres profesionales feministas, buscaban dar cuenta de la condición y situación de las mujeres y denunciar el autoritarismo y el patriarcado presentes en la vida nacional $y$, en los espacios privados.

En 1978 se creó el Círculo de Estudios de la Mujer bajo el alero de la Academia de Humanismo Cristiano, el que a través de la realización de talleres, la difusión de boletines, organización de debates, permitió que convergieran hacia allí diversas organizaciones de mujeres y grupos feministas que poco a poco, además de la denuncia a la dictadura y la defensa de los derechos humanos, comenzaron a debatir diversos temas sobre la familia, la sexualidad, salud reproductiva y divorcio, entre otros. Para esa época, ya destacaban los trabajos de Julieta Kirkwood (1986-1987), investigadora de la Facultad Latinoamericana de Ciencias Sociales (FLACSOChile), pionera de los estudios de mujer y género en Chile y, destacada feminista, que a través de su obra logró unir el activismo político con la reflexión teórica feminista.

Pese al encierro que generó la dictadura, a través de encuentros internacionales las mujeres se conectaban con feministas de otros países, con mujeres chilenas en el exilio y, con organizaciones feministas y de mujeres de otros lugares, lo cual permitió compartir avances y bibliografías que alimentaron el conocimiento sobre la realidad de diversos tipos de mujeres, además de ponerse al día y participar en los debates que iban surgiendo entre las feministas.

El avance de posturas feministas y reivindicaciones respecto al derecho de las mujeres, a tomar decisiones sobre sus vidas y sus cuerpos, generó tensiones con los sectores más conservadores de la Iglesia Católica los que, a fines de 1983, pasaron a la ofensiva ante el avance de las mujeres y las ideas feministas ${ }^{7}$. En 1984 la Academia de Humanismo Cristiano cede a las presiones y expulsa al Círculo de Estudios de la Mujer, por tocar temas incómodos e indeseables para Iglesia. A raíz de esta situación, surge el Centro de Estudios de la Mujer, CEM, y La Casa de la Mujer La Morada. El primero, más dedicado a la investigación de la situación de las mujeres y, la segunda, con un carácter más político feminista, aunque también desarrolló investigación.

El Centro de Estudios de la Mujer, fundado en abril de 1984 por un grupo multidisciplinario de sociólogas, economistas, antropólogas y 
geógrafas, se constituyó en un importante lugar para el desarrollo de investigaciones que tenían como objetivo profundizar en diferentes aspectos relacionados con las vidas de las mujeres, fueran éstas urbanas o rurales, pobladoras o indígenas (Montecino \& Rebolledo,1995). Las inserciones laborales, las mujeres y la educación, la salud, la participación política y las identidades, fueron algunos de los temas abordados y, sobre los cuales se publicaron diferentes libros que visibilizaron los trabajos y actividades de mujeres que no estaban presentes en los estudios realizados hasta la fecha, a las que se les dio voz y un lugar ${ }^{8}$. Paralelamente, se hizo un diagnóstico sobre el mundo de las mujeres, donde se abarcaba temas de trabajo, salud, educación, identidad, participación política, etc. (Centro de Estudios de la Mujer, 1988). La investigación desarrollada en diferentes centros de estudios y al interior de las universidades, por iniciativa de algunas académicas vinculadas a ONG, posibilitó ir constituyendo una base de información importante que permitió sentar las bases para el diseño del programa que, posteriormente -con el retorno a la democracia-, dio origen al SERNAM, Servicio Nacional de la Mujer. A modo de ejemplo, entre los años 1985 y 1993 (Gysling, 1995) se consigna la existencia de 243 trabajos de investigación sobre salud reproductiva, de las cuales el $58,2 \%$ se había producido en las universidades (sumando las tesis de grado). Lo cual daba cuenta de que la capacidad investigativa en ese período se distribuía de manera similar entre universidades y ONG, aunque en el caso de las ONG se trataba básicamente de investigación aplicada.

Además del CEM, que centra su accionar en temas de mujer y trabajo, otros lugares en los cuales se realizaba investigación sobre mujeres, era en la
FLACSO, el Instituto de la Mujer -cuyas líneas centrales son la participación ciudadana de las mujeres y los derechos humanos-, CORSAPS e ICMER, preocupados de temas de salud reproductiva. El año 1990, el equipo especializado en temas de ruralidad y cultura dentro del CEM, crea el Centro de Estudios para el Desarrollo de la Mujer (CEDEM) que se especializa en investigación sobre mujeres campesinas, temporeras e indígenas. Un papel importante en la creación y sustento de estos centros, lo tuvieron agencias internacionales de cooperación al desarrollo, las cuales propiciaron su crecimiento.

En la década de los ochenta, fueron las ONG y los centros de estudios extrauniversitarios, los que desarrollaron más la investigación, en muchos casos fuertemente ligada a la acción, respecto a la situación de las mujeres en Chile. Las universidades, donde las ciencias sociales y humanidades estaban en receso obligado, no fueron el espacio de surgimiento de los estudios de la mujer, aunque estuvieron presentes gracias al esfuerzo individual de algunas pocas académicas comprometidas con el feminismo y, especialmente, por el interés de las estudiantes; así, de la revisión de tesis de grado de las áreas de humanidades y ciencias sociales de las universidades de Chile, Católica, Diego Portales, entre otras (Palacios,1995), se evidencia que entre las estudiantes existía -ya en los años ochenta- un interés por profundizar en la realidad de las mujeres, aunque los enfoques teóricos no fueran los de género y los conceptos tampoco fueran, necesariamente, los acuñados por los estudios de la mujer.

Con el retorno de la democracia, las universidades gradualmente comenzaron a incorporar estos conocimientos que se habían desarrollado fuera de sus espacios. 
Uno de ellos fueron los estudios de mujer y género, los que gracias a la acción de mujeres académicas, a la presión de las estudiantes y, en algunos casos, al financiamiento de agencias de cooperación internacional ${ }^{9}$, poco a poco fueron permeando la necesidad de darles un lugar dentro de la academia, especialmente en las facultades de Ciencias Sociales y Filosofía y Humanidades, a los que posteriormente se agregaron facultades de ciencias médicas y salud. En estos esfuerzos de instalación hubo colaboraciones importantes entre ONG y Universidades, es el caso del seminario "Mujer y Antropología" organizado por el CEDEM y la Facultad de Ciencias Sociales de la Universidad de Chile en 1992 (Montecino \& Boissier, 1993). Así, para el año 1994, se contabilizaban seis programas de estudios de género y mujer, en universidades chilenas ${ }^{10}$, la mayoría de ellas estatales ${ }^{11}$, que habían comenzado sus actividades a comienzos de la década de los noventa. Además, se constató en Santiago la existencia de cursos e investigaciones de género y mujer en cuatro facultades de la Universidad de Chile (Ciencias Sociales, Filosofía y Humanidades, Medicina, Derecho y Ciencias Económicas); por su parte, en la Universidad Católica se dictaron cursos de género en el departamento de Psicología, en Trabajo Social e Historia. Además en la UMCE, UTEM, ARCIS, Diego Portales, Universidad Academia de Humanismo Cristiano, Universidad Bolivariana, Universidad Central, existía al menos un curso de género o mujer (Montecino \& Rebolledo, 1995: Anexo 1). Cuatro años después, en 1998, en ocho universidades santiaguinas se dictaban treinta y siete cursos de género y mujer en pregrado ${ }^{12} y$, ocho programas de postítulo.

La mayor parte de los cursos se dictaban en carreras de pregrado con carácter de electivos y, obedecían más al compromiso y entusiasmo de quienes los dictaban, que a la voluntad de las universidades de incorporar estos temas de manera formal dentro de sus mallas curriculares, lo que explica la paulatina desaparición de muchas de estas iniciativas hacia el final del siglo $X X X^{13}$. Los programas universitarios de estudios de género estaban constituidos inicialmente por feministas que ya estaban en las universidades $y$, por otras, que proviniendo de una ONG contaban con abundante investigación y publicaciones que daban cuenta de su experticia en los temas de género, lo que les permitió diseñar programas de cursos que cumplieran con las exigencias disciplinarias y ganar concursos de cargos. En otros casos, fueron mujeres retornadas del exilio con experiencia en el feminismo y en el mundo académico fuera del país. Todas ellas junto a entusiastas tesistas y jóvenes ayudantes, muchas de las cuales hoy son académicas que continúan trabajando en estudios de género al interior de diversas universidades, fueron dando vida a estos programas y a partir de la reflexión teórica y, metodológica, se fue avanzado hacia la indagación en nuevas temáticas, por ejemplo, la construcción de identidades masculinas y homosexuales, lo que permitió abrir el cerrado círculo anterior de mujeres estudiando mujeres, al incorporarse estudiantes hombres que actualmente siguen trabajando en estos temas. Por otra parte, se renovaron las perspectivas de análisis de temas como la sexualidad que antes se habían abordado desde las ciencias médicas, desde las patologías o disfuncionalidades y, que se vieron enriquecidas con indagaciones sobre comportamientos y construcción de identidades sexuales (Lamadrid \& Muñoz, 1996).

Pese al auge detectado en la década de los noventa, de los cursos e investigaciones sobre 
mujer y género, las dificultades para su instalación y posterior consolidación en los espacios universitarios han sido difíciles, tanto por las reticencias del medio académico a incorporar nuevas perspectivas de análisis -más cuando son críticas del conocimiento instalado como tradición disciplinaria-, como por problemas de financiamiento ${ }^{14}$ de las propias universidades, así como por las características que asumen los fondos concursables. Entre 1982 y 1995, de 4871 proyectos aprobados por el Fondo Nacional de Desarrollo Científico y Tecnológico (FONDECYT), solo veinticinco proyectos, es decir el $0,5 \%$, correspondían a estudios de mujer y género, lo que se explica entre otras cosas por la definición disciplinaria de las áreas de conocimiento establecidas por el concurso y, por las resistencias a abrirse a temáticas nuevas y a otras perspectivas para afrontar la realidad. Esto se hace evidente en que muchas de las investigaciones propuestas entre 1995 y 1996 al Fondo de Investigación para Estudios de Género, creado gracias a la donación de un fondo sueco que luego fue discontinuado, se ubicaban como "sociología", aunque los equipos estaban conformados por investigadores/as responsables provenientes de otras áreas de las ciencias sociales; lo que hace pensar que se asocia sociología a ciencias sociales (Rebolledo \& Donoso, 1998) $)^{15}$.

A partir del retorno a la democracia, las ONG y centros de estudios de la mujer privados, vieron reducirse de manera considerable su financiamiento, lo cual les obligó a orientar los temas de sus investigaciones a la demanda hecha por el SERNAM de estudios específicos para diseñar políticas públicas, compitiendo por esos recursos con los programas y centros universitarios; otras ONG desaparecieron gradualmente. El tipo de investigación se caracterizó por ser investigación aplicada, que asumía la forma de consultoría donde las temáticas a estudiar eran delimitadas desde el Estado, lo cual fue debilitando las posibilidades de una reflexión propia, revisión de conceptos, proposición de nuevas categorías de análisis, análisis de otros sectores sociales, etc., lo que redundó en un cierto estancamiento teórico.

Por su parte, los centros y programas de género universitarios que lograron consolidarse y sostenerse en estos veinte años (CIEG y CEGECAL de la Universidad de Chile) han logrado mantener una mayor libertad en la definición de sus agendas de trabajo académicas (en actividades de docencia, investigación y extensión) abriendo sus campos de reflexión e interlocución a los estudios queer, los estudios poscoloniales y estudios étnicos, gracias a la mayor autonomía que posibilitan los espacios académicos para debatir nuevos temas, para invitar a académicos de otras universidades extranjeras y a la permanente exigencia de los estudiantes, especialmente los de posgrado, por incrementar su formación teórica y metodológica con los debates más actuales. No obstante, la cada vez mayor presión de las universidades por las exigencias de la docencia y los limitados fondos concursables han ido estrechando esa autonomía y, muchas veces la definición de los temas de investigación queda en manos de equipos o académicas individuales que cuentan con el currículo para ganarlos, así como en la decisión de los tesistas.

Actualmente, la investigación realizada en centros de estudio privados, por ejemplo, el CEM, CEDEM o HUMANAS, es temáticamente más acotada que aquella que se desarrolla en los espacios universitarios, en algunos casos por tratarse de investigación aplicada y en otros por mostrar cierta especialización respecto a los sectores con los que se trabaja ${ }^{16}$. 
Por su parte, los centros y programas de género universitarios ya consolidados, después de dos décadas de funcionamiento en los espacios universitarios, luego de una primera etapa de levantamiento de información y análisis respecto al desarrollo de estudios de género, sus aportes epistemológicos, tipo de investigación realizada y docencia dictada, desarrollo de la carreras académicas de las mujeres comparativamente con sus pares masculinos, han pasado a la fase de implementación de propuestas políticas, que apuestan a modificar las inequidades existentes al interior de los espacios universitarios ${ }^{17}$.

A partir del 2010, con los cambios introducidos en las prioridades de SERNAM bajo el gobierno de Sebastián Piñera E., y en algunos casos, por su mayor cercanía con organizaciones no feministas, como Comunidad Mujer o Mujeres Empresarias, varias investigadoras de las ONG de la década de los noventa, migraron hacia las universidades, especialmente privadas, ya sea para la dictación de cursos, realización de investigaciones o creación de centros $^{18}$.

Es de destacar que pese a la institucionalización de los estudios de género y mujer en las universidades, a través de la creación de programas, centros o núcleos, lo que de una u otra manera tiende a imponer las lógicas académicas, se ha logrado mantener un vínculo entre centros de estudios extrauniversitarios y los que están insertos en la educación superior, a diferencia de lo ocurrido en otros países donde se produjo un relativa autonomización de los programas y estudios de género presentes en las universidades, respecto al movimiento feminista y a los centros de estudio privados, dadas las exigencias propias de las disciplinas y la vida académica. Ello, gracias a la presencia de mujeres que se mueven en ambos espacios, lo cual ha posibilitado la realización de investigaciones conjuntas en algunos casos y, la circulación de estudiantes y tesistas en ambas direcciones. Jóvenes profesionales de ONG dedicadas a la investigación de género, suelen concurrir a los programas de Magíster de Género' ${ }^{19}$ para completar su formación y, por otra parte, estudiantes de pregrado y posgrado que realizan sus tesis sobre temas de género, se vinculan a las investigaciones realizadas en los centros privados, ya sea en calidad de ayudantes, tesistas o investigadoras jóvenes.

\section{A modo de conclusiones}

A la hora de hacer un balance de los estudios de mujer y género, vemos avances importantes, hay centros y programas privados y universitarios de reconocido prestigio; se cuenta en la actualidad con un cúmulo importante de estudios en los cuales se ha logrado además profundizar en teorizaciones y propuestas desde una perspectiva latinoamericana, visible en las bibliografías utilizadas en los cursos ${ }^{20}$, en los marcos conceptuales y categorías usadas en las investigaciones; existe un Magíster con dos menciones, consolidado después de 13 años de funcionamiento; a nivel de doctorados son frecuentes los/as estudiantes que realizan sus investigaciones de tesis en temas de género, además de las que se realizan en el pregrado de diversas carreras. Por otra parte, hay un reconocimiento social a estos avances, visibles en la existencia de una Cátedra UNESCO de género en la Universidad de Chile y, por primera vez, una mujer especialista en estudios de género obtuvo, en el 2013, el Premio Nacional de Ciencias Sociales ${ }^{21}$. Sin embargo, las deudas y desafíos pendientes, aun son muchos. 
Entre las deudas está el lograr un reconocimiento real-traducido en financiamientos y en un reconocimiento curricular que incorpore la perspectiva de género en todos los campos del saber- en los espacios universitarios, porque como plantea Thúren respecto a la antropología:

\begin{abstract}
“(...) como el género es un principio organizativo central en todas las sociedades conocidas, su comprensión es fundamental para la comprensión de cualquier otro fenómeno social. La antropología del género dista todavía mucho de ser un tema tan obligatorio para los manuales, para los profesores y para los estudiantes, como por ejemplo el parentesco o el estudio de símbolos. Pero es olvidada como quien no quiere la cosa. Es difícil para el antropólogo de hoy argumentar seriamente por su exclusión. Eso por lo menos se ha conseguido (Thúren, 2011)".
\end{abstract}

Algunos de estos desafíos no son exclusivos de los estudios de género, ya que, como señala Tarrés:

"Los estudios de género al proponer lógicas discursivas surgidas en los márgenes o narrativas e interpretaciones fragmentarias, no acabadas, definidas como "una obra abierta" a la crítica, a las relecturas o a las reinterpretaciones, han planteado un desafío a la idea de verdad, de prueba o verificación contundente y única a la universalidad del conocimiento. En efecto, desde el momento en que supuestos como la diversidad, la diferencia o la relatividad del conocimiento debido a su carácter construido e histórico, revelan la fragilidad e inconsistencia que los paradigmas tradicionales ofrecen para definir la realidad, la naturaleza humana o los modos de acceder al conocimiento, nos enfrentamos, ahora todos, y no solo la comunidad dedicada a los estudios de género, a la necesidad de elaborar paradigmas que integren los nuevos supuestos (Tarrés, 1999: 20)".
Tal vez uno de los mayores méritos de los estudios de género, además de visibilizar a la mitad de la humanidad, haya sido cuestionar los modelo hegemónicos de conocimiento desnudando de paso el poder que ha ejercido desde allí una comunidad determinada, etno y andro centrada para dar por sentada, natural y universal su forma de ver el mundo y las relaciones sociales. Este cuestionamiento permite interrogar a las diferentes disciplinas, dando lugar a la diversidad y la diferencia, criterios que en un mundo globalizado y cambiante son requisitos básicos para el análisis e interpretación en la investigación. Los estudios de género han expandido y enriquecido el campo, abriéndose a nuevos temas como la masculinidad y las homosexualidades; pero a la vez han impulsado el trabajo interdisciplinario, que es necesario seguir profundizando, en el entendido que la fragmentación de los saberes y la falta de perspectivas holísticas es inconducente para dar cuenta de la complejidad sociocultural de la sociedad actual.

A los aportes epistemológicos hechos por los estudios de la mujer y género, hay que agregar las contribuciones realizadas en cuanto a las metodologías de trabajo. Así, por ejemplo, la ruptura de la diferenciación sujeto-objeto, usada tradicionalmente por el paradigma positivo, dando voz a los sujetos/as investigados/as; o bien la utilización de diversas fuentes, no siempre aprovechadas en toda su potencialidad por los investigadores de la realidad social, se constituyen en contribuciones importantes al conjunto de las ciencias sociales.

Por otra parte, más allá del uso y abuso que se ha hecho de la biología para encubrir y, justificar desigualdades y asimetrías sociales de diverso orden, naturalizándolas, es necesario 
reestablecer un diálogo con ella, para no caer en el fundamentalismo de que todo es producto de la cultura. La existencia de una reproducción sexuada, de cuerpos diferentes con potencialidades reproductivas diferentes, sin duda debe producir efectos que la cultura no puede domesticar totalmente lo que nos obliga a revisar y analizar con mayor finura la relación naturaleza cultura. La necesidad de resolver la separación de las ciencias naturales y las humanidades, existente actualmente como fruto de un postulado un tanto arcaico, debería derribar la división disciplinar realizada desde la antigüedad ${ }^{22}$.

Los estudios de mujer y género, gracias a sus vínculos con los movimientos feministas, son una construcción porosa y flexible que en el proceso de crítica y de nuevos hallazgos y, en el diálogo permanente, se ha ido abriendo a nuevos temas y formas de enfrentarlos. Sin duda, su creación relativamente reciente, es un aspecto favorable a esta apertura y capacidad de desplazar fronteras de conocimiento, a buscar nuevos conceptos que permitan dar cuenta de relaciones sociales cada vez más complejas en un mundo globalizado ${ }^{23}$. La revisión crítica de algunos de sus basamentos teóricos, la creación de nuevas categorías que se ajustan de mejor manera a la aprehensión de realidades complejas y cambiantes, permitió el paso de los estudios de mujer a los de género. Hoy los desafíos surgen de otros estudios, que necesariamente entran en diálogo con los planteamientos de los estudios de género, tensionando nuevamente el campo y exigiendo dar un paso más allá. En palabras de Joan Scott en su historia del feminismo:

"El nuevo territorio recientemente seguro del género y la historia de las mujeres está siendo desfamiliarizado, mientras los estudios queer, los estudios postcoloniales los estudios étnicos (entre otros) nos retan a llevar más lejos las fronteras, a deslizarnos (¿o brincar?) de manera metonímica a terrenos contiguos".

Y tal vez, ese movimiento permite dar el gran salto y cuestionar el modelo existente de universidad.

\section{Notas}

${ }^{1} \mathrm{El}$ androcentrismo se refiere al "Enfoque de un estudio, análisis o investigación desde la perspectiva masculina únicamente y la utilización posterior de los resultados como válidos para la generalidad de los individuos, hombres y mujeres" (Sau, 1989: 45).

${ }^{2}$ En 1984, Chandra Mohanty, en su análisis sobre la construcción de las mujeres del tercer mundo realizada por las feministas occidentales, las enmarca en el discurso colonial, señalando que "las prácticas del feminismo académico (ya sea de lectura, escritura, crítica o textual) están inscritas en las relaciones de poder, relaciones a las que se enfrentan, resisten o, quizás, incluso respaldan implícitamente. No existe, por supuesto la academia apolítica" (Mohanty, en Suárez \& Hernández, 2008:2).

${ }^{3}$ Para un análisis de los aportes de las mujeres afrodescendientes a la teoría feminista véase Curiel (2007).

${ }^{4}$ Esta discusión ha sido llevado adelante con especial vigor por parte de las feministas antirracistas (cfr. Espinoza: 2009).

${ }^{5}$ Como ocurrió en los EEUU (Stimpson, 1986), Inglaterra y otros países europeos (Montecino, 1995).

${ }^{6}$ Es de destacar que a finales de los años 60 y comienzos de los 70 , algunas destacadas investigadoras que han hecho importantes aportes a los estudios de mujer y género, fueron investigadoras o alumnas de FLACSO Chile. Es el caso de Teresita de Barbieri, Orlandina de Oliveira y Julieta Kirkwood.

${ }^{7}$ Así, el 2 de diciembre 1983, Tradición Familia y Propiedad, publica en El Mercurio un inserto titulado "EN NOMBRE DE LA FE HAY QUIENES PRETENDEN LLEVARNOS A UN RÉGIMEN ATEO, SANGUINARIO Y DESPÓTICO. UN RÉGIMEN TÍTERE DE MOSCÚ". 
Tradición Familia y Propiedad documenta ampliamente esta realidad y pide a Dios, a la Virgen y a la Iglesia remedios para esta situación, porque Chile no quiere sucumbir". En el punto 4ำ subtitulado "Academia de Humanismo Cristiano promueve el hedonismo más impúdico", se planteaba que: "El Círculo de Estudios de la Mujer de la Academia de Humanismo Cristiano, dependiente del arzobispado de Santiago publica revistas, que a la par de ser socialistas en el plano político, son inmorales en el plano de las costumbre. A lo largo de sus páginas se incentiva el orgullo y la sensualidad, que son respectivamente las causas del igualitarismo y del libertinaje." (En Gaviola et.al. 1994).

${ }^{8}$ Resultado de algunas de esas investigaciones son: Historias testimoniales de mujeres del campo (Valdés et. al. 1982), Oficios y trabajos de las mujeres de Pomaire (Valdés \& Matta, 1986), Mujeres de la Tierra (Montecino, 1984); Andar andando. Testimonios de mujeres del sector forestal (De León, 1986); Trabajo doméstico remunerado: conceptos, hechos datos (Todaro \& Gálvez, 1987).

${ }^{9}$ Así, por ejemplo, el PIEG, Programa Interdisciplinario de Estudios de Género de la Facultad de Ciencias Sociales y el CEGECAL, Centro de Estudios de Género y Cultura de América Latina, de la Facultad de Filosofía y Humanidades, recibieron apoyo de la Fundación FORD, la que además promovió la apertura del programa de estudios de género en la Pontificia Universidad Católica de Lima en Perú.

${ }^{10}$ En dichos programas además de la docencia se desarrollaba investigación y actividades de extensión.

${ }^{11}$ EI PIEG y CEGECAL en las Facultades de Ciencias Sociales y de Filosofía y Humanidades de la Universidad de Chile el Programa de estudios de la Mujer en la Universidad de Concepción en la Facultad de Filosofía; el CECODEM, en la Universidad de Santiago en la Facultad de Ciencias Médicas; el Programa Interdisciplinario de Educación y Género en la Universidad de La Serena; el Programa de Estudios de Género de la Universidad Santos Ossa (Montecino \& Rebolledo, 1995).

${ }^{12}$ De ellos tres se dictaban carreras de Antropología. Se trataba de los cursos de Antropología de la Mujer, en la Universidad Academia de Humanismo Cristiano, Sociología de la Mujer en Medicina de la Universidad de Chile y Antropología del género en la Universidad de Chile (Montecino \& Obach, 1998).
${ }^{13}$ Esto no significa que los temas de género y mujer no sigan ocupando un lugar en las cátedras universitarias, pero han ido derivando hacia nuevas modalidades, programas de postgrado (magíster y diplomados). Y se mantienen aún cursos de carácter electivo en algunas universidades a nivel del pregrado, además de Seminarios de tesis o la introducción de una perspectiva de género en cursos interdisciplinarios.

${ }^{14}$ Hay que recordar que la instalación de los estudios de género en las universidades, se da en un momento de creciente privatización de la educación superior, en un contexto neoliberal en que la docencia profesionalizante fue adquiriendo cada vez mayor importancia como forma de financiamiento de las universidades, sumado a la reducción del presupuesto para la investigación.

${ }^{15}$ Los diseños metodológicos de la investigación de mujer y género son de corte cualitativo, cuantitativo y una combinación de ambos. Los estudios cualitativos, con aplicación de sus diferentes técnicas -entrevistas en profundidad, observación participante, grupos focales, historias de vida, genealogías-se usan especialmente cuando se trata de temas en los cuales se busca profundizar en la construcción de identidades, relaciones de género y otros aspectos relacionados con las prácticas culturales y cotidianas. Los estudios cuantitativos son más utilizados en investigaciones sobre trabajo, salarios y participación política y los estudios mixtos, donde se recurre al uso de datos cualitativos y cuantitativos, son aquellos sobre salud, conciliación trabajo-familia, entre otros.

${ }^{16}$ Así el CEM se orienta fundamentalmente al trabajo de corte sociológico y económico respecto a temas de trabajo y salud en zonas urbanas y el CEDEM trabaja en sectores rurales con aproximaciones más cercanas a la antropología. Por su parte Humanas tiene un marcado énfasis temático en derechos humanos.

${ }^{17}$ Es el caso de la Universidad de Chile, donde la Comisión de Igualdad de Género, conformada por académicas de diversas Facultades e Institutos y bajo el alero de la Vicerrectoría de Extensión, ha hecho un importante estudio sobre la realidad de género en dicho centro de educación superior (Universidad de Chile).

${ }^{18}$ Entre las universidades receptoras de este tipo de investigadoras se encuentra la Universidad Diego Portales, 
la Universidad Alberto Hurtado y la Universidad Santo Tomás, entre otras.

${ }^{19}$ Desde el año 2000 existe el magíster de Género y Cultura en la Universidad de Chile, el que cuenta con una mención en Ciencias Sociales y otra en Humanidades.

${ }^{20}$ Las bibliografías más recurrentes en los cursos de género tienen un referente importante en la producción latinoamericana donde destacan, entre otros, los nombres de Marta Lamas, Teresita de Barbieri, Sonia Montecino, Milagros Palma, Nieves Rico, Magdalena León, Jeanine Anderson.

${ }^{21}$ Se trata de la antropóloga Sonia Montecino.

${ }^{22}$ A modo de ejemplo, se puede señalar la imbricación entre los adelantos científico-tecnológicos y las humanidades y ciencias sociales, a partir del ejemplo de las innovaciones introducidas en las últimas décadas en las técnicas reproductivas. La fertilización asistida, los bancos de óvulos y espermios, la implantación de estos en vientres de alquiler, no solo ponen en cuestión la necesidad de la existencia de una pareja de hombre y mujer para reproducir la especie, y obligan a redefinir los conceptos de maternidad y paternidad, también movilizan temas éticos, sociales y culturales; por lo tanto, la aproximación a problemas de este orden exige la cooperación de disciplinas diversas para su abordaje.

${ }^{23}$ Así, por ejemplo, desde el feminismo de color y tercer mundista se ha propuesto diversos términos: interconexión, entrelazamiento, interseccionalidad, etc., para dar cuenta de cómo opera la opresión de manera múltiple sobre muchas mujeres.

\section{Referencias bibliográficas}

Bonder, G., ed. (1998). Estudios de la mujer en América Latina, Secretaría General de la OEA, Colección INTERAMER 56, Serie Cultural, Washington.

Bonder, G. (1999). "Género y Subjetividad: avatares de una relación no evidente" en Género y Epistemología, Mujeres y disciplinas, Sonia Montecino y Alejandra Obach, compiladoras, Santiago: LOM.

Centro de Estudios de la Mujer (1988). Mundo de Mujer, Continuidad y cambio. Santiago: Ediciones CEM. Curiel, O. (2007) "Los aportes de las afrodescendientes a la teoría y práctica feminista. Desuniversalizando el sujeto "mujeres". En María Luisa Femenías (Comp.) Perfiles del feminismo Iberoamericano vol. III, Buenos Aires: Catálogos.

De León, K. (1986). Testimonios de Mujeres del sector forestal, Santiago: Ediciones CEM.

Espinoza, Y. (2009). "Etnocentrismo y colonialidad en los feminismos latinoamericanos: complicidades y consolidación de las hegemonías feministas en el espacio transnacional". Revista Venezolana de Estudios de la Mujer, julio/diciembre 2009, vol 14, № 33, pp.37-54.

Gaviola, E., Largo, E. \& Palestro, S. (1994). Una historia necesaria: mujeres en Chile 1973-1990. Santiago: Aki\& Ahora Ltda.

Gysling, J. (1995). La investigación social en salud reproductiva en Chile. Panorama al inicio de los noventa, PIEG-Universidad de Chile- FLACSO. Santiago.

Kirkwood, J. (1986). Ser política en Chile. Las feministas y los partidos. Santiago: FLACSO.

Kirkwood, J. (1987). Feminarios. Santiago: Ediciones Documentas/Mujer.

Lamadrid, S. \& Muñoz, S. (1996). La investigación social en sexualidad en Chile, 1984-1994, Santiago: PIEG- Universidad de Chile, Serie Apuntes Docentes. Lamas, M. (1996). ¿Identidades?, Editorial Debate Feminista, año 7, vol.14, octubre Identidades, México. Mohanty, Ch. (2008). "Bajo los ojos de occidente. Academia Feminista y discurso colonial". En Suárez, L. \& Hernández, A. (eds). Descolonizando el feminismo: Teorías y prácticas desde los márgenes. Madrid: Ediciones Cátedra.

Mona, E. \& Leacock, E. (1980). Women and colonization. Anthropological perspectives. New York: Preage, Berlin Publishers Book.

Montecino, S. (1984). Mujeres de la tierra, Santiago: Ediciones CEM.

(1991). Madres y Huachos: alegorías del mestizaje chileno. Santiago: CEDEM-Cuarto propio. (1995). "De la mujer al género: Implicancias Académicas y teóricas". En Montecino, S. \& Rebolledo, L. (Comps.) Mujer y género, nuevos saberes en las Universidades chilenas. Santiago: Bravo editores. Montecino, S. \& Boissier, M.E. (1993). Huellas, Seminario Mujer y Antropología, Santiago: Ediciones CEDEM. 
Montecino, S. \& Obach, A. (1998). "De la descripción al cambio en las relaciones de género. Breve análisis de los currículos de mujer y género en las universidades chilenas". En Montecino, S. \& Obach, A. (Comps.), Género y Epistemología, Mujeres y Disciplinas. Santiago: PIEGUniversidad de Chile.

Ortner; S. (1979). “¿Es la mujer respecto al hombre lo que la naturaleza con respecto a la cultura?" En Antropología y feminismo. Barcelona.

Palacios, P. (1995). Listado de Tesis sobre mujer y género en algunas universidades chilenas, Santiago: PIEG- Universidad de Chile.

Rebolledo, L. \& Donoso, C. (1998). "Disciplina o Interdisciplina: Balance preliminar del fondo de género CONICYT Chile". En Montecino, S. \& Obach, A. (Comps.) Género y Epistemología, Mujeres y Disciplinas. Santiago: PIEG-Universidad de Chile. Serie Documentos.

Sau, V. (1989). Diccionario ideológico feminista. Barcelona: Icaria Editorial.

Scott, J. Historia del feminismo. Disponible en: $\underline{w w w . r a c o .}$ index/.php/HojasWarmi/article/viewFile/179370/231865 Fecha de consulta: 25 de enero de 2014.

Stimpson, C. (1986). Women studies in the United States. New York: Report FORD Foundation.

Stolcke, V. "Antropología del género. El Cómo y el porqué de las mujeres". Cholonautas. Biblioteca virtual de Ciencias Sociales. Disponible en: www.cholonautas. edu.pe Fecha de consulta: 25 de enero de 2014.

Tarrés, M.L. (1999). Palabras inaugurales en Encuentro de Universidades de Latinoamérica y del Caribe. En Montecino S. \& Obach, A. (Comps.) Género y Epistemología, Mujeres y disciplinas en Género y Epistemología, Mujeres y disciplinas, Santiago: LOM, 1999.

Todaro, R. \& Gálvez, T. (1987). Trabajo doméstico remunerado. Conceptos, hechos, datos. Santiago: Ediciones CEM.

Thúren, B-M. (s.f). "Del sexo al género: Un desarrollo teórico. 1970-1990". Disponible en: http://bmthuren.files.wordpress.com/2011/12/ delsexoalgecc81neroantropologicc81a.pdf Fecha de consulta: 25 de enero de 2014.

Valdés, X. \& Matta, P. (1986). Oficios y trabajos de las mujeres de Pomaire. Santiago: Ediciones CEM.
Valdés, X. (1992). Los nudos de la modernización agraria. Santiago: Ediciones CEDEM.

Valdés, X. et.al. (s.f.), Historias Testimoniales de las mujeres del campo. Santiago: Círculo de estudios de la Mujer- Academia de Humanismo Cristiano-PEMCl. Universidad de Chile. (2013). Del biombo a la cátedra: igualdad de oportunidades de género en la Universidad de Chile. Comisión de Igualdad de Género de la Universidad de Chile. 